\title{
Desenvolvimento inicial de plantas de mandioca em sistema de cultivo mínimo e preparo convencional com diferentes massas de coberturas vegetais
}

\author{
Initial development of cassava plants in a minimum cultivation system \\ and conventional preparation with different types of vegetative cover
}

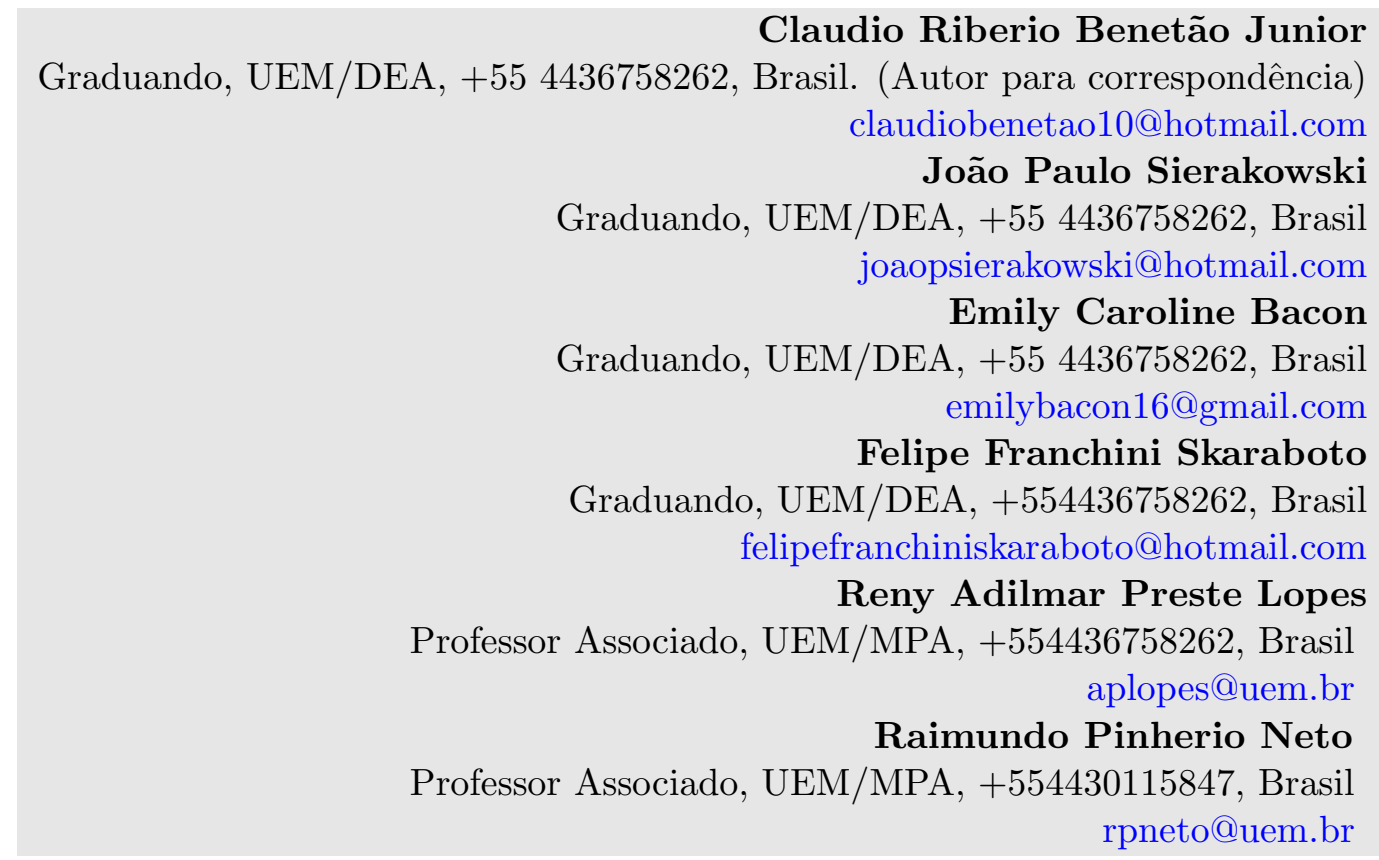

\section{Resumo}

Os solos arenosos com cultivo de mandioca em sistema de preparo convencional sofrem intenso revolvimento, que pode acarretar desestruturação e adensamento do solo. O solo da região noroeste do Paraná é originário do Arenito Caiuá, apresentam na sua textura teor de areia oscilando entre $85 \%$ a $90 \%$, possuindo níveis baixos de fósforo, potássio, cálcio, magnésio e baixo teor de matéria orgânica, geralmente com alta susceptibilidade à erosão. O experimento foi realizado no Campus do Arenito, Universidade Estadual de Maringá, em Cidade Gaúcha. Utilizou-se a variedade de mandioca Fécula Branca sobre preparo convencional e cultivo mínimo, com quatro composições de coberturas vegetais: aveia preta comum com crotalária juncea $(\mathrm{AvC})$; aveia preta comum com milheto comum $(\mathrm{AvM})$, aveia preta comum com crotalária juncea e milheto comum (AvCM) e aveia preta comum (Av). O desenvolvimento inicial das plantas de mandioca e massa seca das coberturas vegetais foram influenciados pelos sistemas de preparo do solo. $\mathrm{O}$ cultivo mínimo apresentou menores valores de diâmetro de colmo, altura de plantas e brotação de manivas. Enquanto que o preparo convencional, apresentou menor número de plantas e maior espaçamento entre 
plantas em parcelas sob aveia preta em sucessão de crotalária juncea com milheto e parcelas somente com aveia preta. A cobertura vegetal aveia preta sucessão com crotalária juncea, sob cultivo mínimo e aveia preta sucessão com crotalária juncea e milheto comum, sob preparo convencional, apresentaram os maiores valores de massa seca.

Palavras-chave: plantio de mandioca; manejo do solo; desenvolvimento de plantas.

\begin{abstract}
The sandy soils with cassava cultivation in conventional preparation system suffer intense revolving, which can lead to destructuring and soil thickening. The soil in the northwestern region of Paraná originates from the Caiuá Sandstone, have in its texture sand content ranging from $85 \%$ to $90 \%$, with low levels of phosphorus, potassium, calcium, magnesium and low organic matter content, usually with high susceptibility to erosion. The experiment was carried out at Campus do Arenito, Universidade Estadual de Maringá, in Cidade Gaúcha. The White Starch cassava variety was used on conventional preparation and minimum cultivation, with four plant cover compositions: common black oat with junceous crotalaria $(\mathrm{AvC})$; common black oat with common millet (AvM), common black oat with junceous crotalaria and common millet $(\mathrm{AvCM})$ and common black oat $(\mathrm{Av})$. The initial development of cassava plants and dry mass of plant coverings were influenced by soil preparation systems. The minimum crop showed lower values of thatch diameter, plant height and manioc sprouting. While conventional preparation, there were fewer plants and greater spacing between plants in plots under black oats in succession of crotalaria juncea with millet and plots with black oats only. The plant cover black oat succession with junceous crotalaria under minimal cultivation and black oat succession with junceous crotalaria and common millet under conventional preparation presented the highest values of dry mass.
\end{abstract}

Keywords: cassava planting; soil management; plant development

\section{Introdução}

Os solos arenosos com cultivo de mandioca em sistema de preparo convencional sofrem intenso revolvimento, acarretando a desestruturação e o adensamento do solo. O solo da região noroeste do Paraná é originário do Arenito Caiuá, apresentam na sua textura teor de areia oscilando entre $85 \%$ a $90 \%$, possuindo níveis baixos de fósforo, potássio, cálcio, magnésio e baixo teor de matéria orgânica, geralmente com alta susceptibilidade à erosão (Oliveira et al., 2000). Segundo Valle et al., (2008), com estudos de melhorias de plantio, variedades e práticas de produção, a mandioca pode chegar a produtividade de até $90 \mathrm{t} \mathrm{ha}^{-1} \mathrm{ano}^{-1}$. O Arenito Caiuá, na sua maioria, é explorado por pecuária, cultura da cana-de-açúcar e mandioca, sendo implantadas sob preparo convencional. $\mathrm{O}$ cultivo mínimo associado ao uso de coberturas vegetais pode proporcionar melhores índices de desenvolvimento das plantas de mandioca, redução do efeito erosivo no solo, melhoria da qualidade física e química quando comparados ao preparo convencional do solo. Diante do exposto, este trabalho teve como objetivo avaliar o desenvolvimento inicial das plantas de mandioca em diferentes sistemas de preparo e cobertura do solo.

\section{Material e Métodos}

O experimento foi realizado no Campus do Arenito, Universidade Estadual de Maringá, em Cidade Gaúcha. Utilizou-se a variedade de mandioca Fécula Branca sobre preparo convencional e cultivo mínimo, com quatro composições de coberturas vegetais. As parcelas experimentais foram compostas por mandioca, plantadas sobre parcelas sob preparo convencional e cultivo mínimo. Nos diferentes sistemas de preparo do solo, as coberturas vegetais foram semeadas no inverno e verão. O milheto comum (Pennisetum americanum) e crotalária juncea (Crotalaria juncea) foram 
semeadas no verão com dosagem de15 $\mathrm{kg} \mathrm{ha}^{-1} \mathrm{e}$ $30 \mathrm{~kg} \mathrm{ha}^{-1}$, a aveia preta comum (Avena strigosa) foi semeada no período de inverno com dosagem de $50 \mathrm{~kg} \mathrm{ha}^{-1}$ na dosagem recomendada segundo Carlos et al., (2006). A massa seca das coberturas vegetais aveia preta (inverno), milheto comum (verão) e crotalária juncea (verão) e a composição milheto comum/crotalária juncea (verão), foram determinadas utilizando-se de quatro amostragens, em cada parcela no verão e no inverno antes do plantio da mandioca. A massa seca das parcelas de aveia preta comum foram somadas à massa seca do milheto comum, da crotalária juncea e à composição milheto comum/crotalária juncea, tendo um total de massa seca anual $\left(\mathrm{t} \mathrm{ha}^{-1}\right)$. A análise das plantas de mandioca foi realizada, em 10 plantas, sendo as amostras coletadas ao acaso nas linhas centrais, 60 dias após o plantio da mandioca. Mensurou-se o diâmetro de colmos (m), altura de plantas $(\mathrm{m})$, número de plantas por metro (planta $\mathrm{m}^{-1}$ ), brotos de manivas por planta (brotos planta $^{-1}$ ), espaçamento entre plantas $(\mathrm{m})$, segundo metodologia descrita em Fialho e Vieira (2011). Os tratamentos experimentais foram compostos por parcelas com AvC (Aveia preta comum com Crotalária juncea); AvM (Aveia preta comum com Milheto comum; AvCM (Aveia preta comum com consorcio Crotalária juncea e Milheto comum; Av (Aveia preta comum) sob os sistemas de preparo do solo convencional (PC) e cultivo mínimo (CM). Os dados foram analisados em esquema fatorial, em faixas, pelo Teste $\mathrm{F}$ e teste de agrupamento de médias Scott-knott a nível de 5\% de probabilidade no programa computacional Sisvar 5.3.

\section{Resultados e Discussão}

Na Tabela 1, são apresentados os resultados de massa seca das coberturas vegetais em preparo convencional e cultivo mínimo. Analisando-se os valores massa seca das coberturas vegetais, verifica-se que as parcelas com a cobertura vegetal $\mathrm{AvC}$ teve maior valor de massa seca no sistema cultivo mínimo, enquanto que AvM e AvCM, destacaram-se em maiores valores de massa seca no sistema convencional.

As parcelas com aveia (Av) tiveram comportamentos semelhantes em ambos os sistemas de preparo do solo. Os valores de massa seca observados para aveia preta são inferiores aos observados por Oliveira, et al., (2000) que avaliando a aveia preta verificaram que produção de matéria seca foi de $4,325 \mathrm{t} \mathrm{ha}^{-1}$ para IAPAR61 e para aveia branca foi $5,914 \mathrm{t} \mathrm{ha}^{-1}$ para variedade IAPAR 96101-B.

No sistema cultivo mínimo, AvC destacou-se em maior valor de massa seca e no sistema convencional, o maior valor de massa seca sobre o solo foi observado nas parcelas com AvCM.

Tabela 1. Massa seca de coberturas vegetais em diferentes sistemas de manejo

\begin{tabular}{llllll}
\hline $\begin{array}{l}\text { Preparo/Cobertura } \\
\text { vegetal }\end{array}$ & $\begin{array}{l}\text { Massa Seca }\left(\mathrm{t} \mathrm{ha}^{-1}\right) \\
\mathrm{AvC}\end{array}$ & $\mathrm{AvM}$ & $\mathrm{AvCM}$ & $\mathrm{Av}$ & Média \\
\hline $\begin{array}{l}\text { Cultivo Mínimo } \\
\text { Convencional }\end{array}$ & $19.281 \mathrm{~A}^{a}$ & $10.172^{B c}$ & $14.810^{B b}$ & $4.095^{A d}$ & $12.090^{A}$ \\
\hline Média & $10.230^{B c}$ & $14.795^{A b}$ & $19.287^{A a}$ & $4.065^{A d}$ & $12.094^{A}$ \\
\hline
\end{tabular}

*AvC $=$ aveia preta comum com crotaláriajuncea; $\mathrm{AvM}=$ aveia preta comum com milheto comum; AvCM = aveia preta comum com crotaláriajuncea e milheto comum; $\mathrm{Av}=$ aveia preta comum. Médias seguidas pela mesma letra, maiúscula na coluna e minúscula na linha, não diferem entre si pelo teste de agrupamento de Scott-knott a nível de 5\% de probabilidade.

Na Tabela 2, são apresentados os resultados Verificou-se entre o sistema de preparo do solo, os das características fisiológicas das plantas de menores valores de diâmetro foram observados nas mandioca em preparo convencional e cultivo mínimo. parcelas AvC e AvCM no sistema cultivo mínimo. 
Em relação as coberturas vegetais, no sistema cultivo mínimo, o maior valor médio de diâmetro foi observado foi nas parcelas com AvM, enquanto que no sistema convencional foi nas parcelas com a cobertura vegetal AvCM. Estudos de Aguiar et al (2009), avaliando o comportamento da parte aérea com diferentes densidades de plantio, verificaram que menores densidades de plantio apresentam melhor desenvolvimento de números de plantas e diâmetro das hastes. A variável altura de plantas apresentou menor valor médiosob o sistema cultivo mínimo, nas parcelas com as coberturas AvC e AvCM.Na análise de coberturas vegetais, em cada sistema de preparo, verificou-se que somente as parcelas com Av apresentaram o menor valor médio de altura de plantas no sistema convencional.

Tabela 2. Características fisiológicas de mandioca em diferentes manejos

\begin{tabular}{|c|c|c|c|c|c|}
\hline \multirow{2}{*}{$\begin{array}{l}\text { Preparo/Cobertura } \\
\text { vegetal }\end{array}$} & \multicolumn{4}{|c|}{ Diâmetro de Colmos $\left(\mathrm{m} \mathrm{10}{ }^{-3}\right)$} & \multirow[b]{2}{*}{ Média } \\
\hline & $\mathrm{AvC}$ & AvM & $\mathrm{AvCM}$ & Av & \\
\hline Cultivo Mínimo & $5.83^{B b}$ & $9.43^{A a}$ & $6.43^{B b}$ & $6.78^{A b}$ & $7.11^{B}$ \\
\hline Convencional & $7.93^{A b}$ & $8.15^{A b}$ & $9.88^{A a}$ & $7.30^{A b}$ & $8.31^{A}$ \\
\hline Média & $6.88^{b}$ & $8.79^{a}$ & $8.15^{a}$ & $7.04^{b}$ & \\
\hline Preparo/Cobertura & \multicolumn{4}{|c|}{ Altura de Plantas $\left(\mathrm{m} 10^{-2}\right)$} & \\
\hline vegetal & $\mathrm{AvC}$ & AvM & $\mathrm{AvCM}$ & Av & Média \\
\hline Cultivo Mínimo & $9.75^{B a}$ & $14.60^{A a}$ & $8.70^{B a}$ & $11.15^{A a}$ & $11.05^{B}$ \\
\hline Convencional & $19.60^{A a}$ & $17.40^{A a}$ & $18^{A a}$ & $12.95^{A b}$ & $16.99^{A}$ \\
\hline Média & $14.68^{a}$ & $16^{a}$ & $13.35^{a}$ & $12.05^{a}$ & \\
\hline Preparo/Cobertura & \multicolumn{4}{|c|}{ Número de Plantas (plantas $10^{-1}$ ) } & \\
\hline vegetal & $\mathrm{AvC}$ & AvM & $\mathrm{AvCM}$ & Av & Média \\
\hline Cultivo Mínimo & $1.45^{A a}$ & $1.75^{A a}$ & $1.50^{A a}$ & $1.60^{A a}$ & $1.58^{A}$ \\
\hline Convencional & $1.75^{A a}$ & $1.65^{A a}$ & $1.35^{A b}$ & $1.30^{A b}$ & $1.51^{A}$ \\
\hline Média & $1.60^{a}$ & $1.70^{a}$ & $1.43^{a}$ & $1.45^{a}$ & \\
\hline Preparo/Cobertura & \multicolumn{4}{|c|}{ Brotação das Manivas (brotos plantas ${ }^{-1}$ ) } & \\
\hline vegetal & $\mathrm{AvC}$ & AvM & $\mathrm{AvCM}$ & $\mathrm{Av}$ & Média \\
\hline Cultivo Mínimo & $1.15^{A a}$ & $1.05^{A a}$ & $1.00^{A a}$ & $1.00^{A a}$ & $1.05^{B}$ \\
\hline Convencional & $1.35^{A a}$ & $1.20^{A a}$ & $1.30^{A a}$ & $1.25^{A a}$ & $1.28^{A}$ \\
\hline Média & $1.25^{a}$ & $1.13^{a}$ & $1.15^{a}$ & $1.13^{a}$ & \\
\hline Preparo do solo/ Cobertura & \multicolumn{4}{|c|}{ Espaçamento entre Plantas (m) } & \\
\hline vegetal & $\mathrm{AvC}$ & AvM & $\mathrm{AvCM}$ & $\mathrm{Av}$ & Média \\
\hline Cultivo Mínimo & $0.73^{A a}$ & $0.69^{A a}$ & $0.63^{A a}$ & $0.75^{A a}$ & $0.69^{A}$ \\
\hline Convencional & $0.54^{A b}$ & $0.63^{A b}$ & $0.75^{A a}$ & $0.89^{A a}$ & $0.70^{A}$ \\
\hline Média & $0.63^{b}$ & $0.66^{b}$ & $0.69^{b}$ & $0.82^{a}$ & \\
\hline
\end{tabular}

*AvC $=$ aveia preta comum com crotaláriajuncea; $\mathrm{AvM}=$ aveia preta comum com milheto comum; AvCM $=$ aveia preta comum com crotaláriajuncea e milheto comum; Av = aveia preta comum. Médias seguidas pela mesma letra, maiúscula na coluna e minúscula na linha, não diferem entre si pelo teste de agrupamento de Scott-knott a nível de $5 \%$ de probabilidade.

Estudos realizados por Lopes, et al., (2015), mostraram que a altura de plantas foi influenciada pelos sistemas de preparo, tipo de hastes e variedades de mandioca. Estes autores identificaram valores inferiores de altura de plantas no sistema CM, em parcelas com Fécula Branca plantadas com haste tipo facão sulcador alado + Disco de corte corrugado frontal (FAD) e haste tipo facão sulcador simples + Disco de corte corrugado frontal (FSD). Menores números de plantas foram observados nas parcelas com Av e AvCM. Para brotação de planta, menores valores foram observados no sistema cultivo mínimo, 
independente da cobertura vegetal utilizada. Para espaçamento entre plantas maior valores foram observados nas parcelas somente com Av. Estudos conduzidos por Irolivea, et al., (1998), estudando o efeito do espaçamento entre plantas e da arquitetura varietal no comportamento vegetativo e produtivo da mandioca, verificaram que cultivares que se ramificaram com espaçamentos menores favoreceram o fechamento precoce das copas, reduziram o ângulo de ramificação e o diâmetro da haste principal e os menores espaçamentos aumentaram o rendimento dos cultivares que não se ramificaram.

\section{Conclusões}

O desenvolvimento inicial das plantas de mandioca e massa seca das coberturas vegetais foram influenciados pelos sistemas de preparo do solo. O cultivo mínimo apresentou menores valores de diâmetro de colmo, altura de plantas e brotação de manivas. Enquanto que o preparo convencional, apresentou menor número de plantas e maior espaçamento entre plantas em parcelas sob aveia preta em sucessão de crotalária juncea com milheto e parcelas somente com aveia preta. A cobertura vegetal aveia preta sucessão com crotalária juncea, sob cultivo mínimo e aveia preta sucessão com crotalária juncea e milheto comum, sob preparo convencional, apresentaram os maiores valores de massa seca.

\section{Referências Bibliográficas}

Aguiar, E. B., Bicudo, S., Curcelli, F., Abreu, M. L., Passini, C. T., Brachtvogel, E. L., Cruz, S. S., 2009. Desenvolvimento da parte aérea da mandioca sob diferentes densidades populacionais em dois tipos de solo. In: XIII Congresso Brasileiro de Mandioca: Inovações e desafios, 2009, Botucatu. Anais... Botucatu: CERAT/UNESP. https://doi.org/10.32905/19833253.2018.10.s14p29
Carlos, J. A. D., COSTA, J. A.; COSTA, M. B. Adubação Verde: do conceito à prática. Piracicaba: ESALQ/DIBD. 2006. 32p. (Série Produtor Rural, $30)$.

Irolivea, E. A. M., Câmara, G. M. S., Nogueira, M. C. S., Cintra, H. S., 1998. Efeito do espaçamento entre plantas e da arquitetura varietal no comportamento vegetativo e produtivo da mandioca. Piracicaba: Scientia Agrícola, v.55, n.2, p. 269-27. https://doi.org/10.1590/s0103-90161998000200016

Fialho, J. F., Vieira, E. A., 2011. Seleção participativa de variedades de mandioca na agricultura familiar. Planaltina: Embrapa Cerrados, $76 \mathrm{p}$

Lopes, R. A. P., Milani, J. L. B., Skaraboto, F. F., Dorne, E. O., Lozano, C. S., Silva, D. R., 2015. Altura de plantas, produtividade e teor de amido de mandioca com diferentes manejos e hastes sulcadoras. In: XVI Congresso Brasileiro de Mandioca/ I Congresso Latino-Americano e Caribenho de Mandioca, 2015, Foz do Iguaçu. Anais... Foz do Iguaçu: CERAT/UNESP. https://doi.org/10.5151/enemp2015-cd-561

Oliveira, E., Medeiros, G. B., Marun, F., Oliveira, J. C., SÁ, J. P. G., Filho, A. C., Kranz, W. M., Silva J. R. N. F., Abrahão, J. J. S., Guerini, V. L., Martin, G. L., 2000. Recuperação de pastagens no Noroeste do Paraná: bases para plantio direto e integração lavoura pecuária. Londrina: IAPAR, 2000, 96p. (Informe de Pesquisa, 134). https://doi.org/10.31692/2526-7701.iicointerpdvagr o.2017.00094

Valle, T. L., Feltran, J. C., Carvalho, C. R. L., 2008. Mandioca para produção de etanol. Campinas: Portal Instituto Agronômico, 16p

La Revista Ingeniería y Región cuenta con la Licencia Creative Commons Atribución (BY), No Comercial (NC) y Compartir Igual (SA)

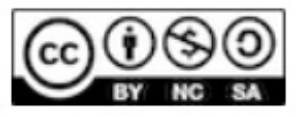

